$>$ Aux yeux du grand public, la «maladie cardia-

La valve aortique

n'a pas révélé

tous ses secrets

Gregor Andelfinger

que » est souvent synonyme de maladie coronarienne. On oublie facilement que le rétrécissement aortique cause à lui seul 100000 opérations et 20000 décès par an en Amérique du Nord. Les enjeux médicaux et sociaux sont de plus en plus importants en raison du vieillissement de la population et de l'augmentation des facteurs de risque pour la progression du rétrécissement aortique. <

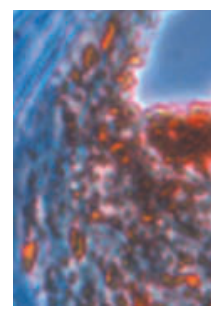

au développement d'approches thérapeutiques efficaces. Ainsi, la découverte que des mutations de Notchl peuvent causer des sténoses aortiques suscite un intérêt particulier [5]. Ce travail est le premier à décrire une cascade étiologique expliquant la calcification aortique au niveau moléculaire, identifiant ainsi une cible thérapeutique potentielle pour la prévention de la progression de la sténose aortique. Compte tenu de ces découvertes, quelles seraient donc les approches les plus prometteuses pour améliorer le diagnostic et le traitement du rétrécissement aortique? Dans ce numéro de médecine/sciences [6] $(\rightarrow)$, le docteur Philippe Pibarot, récipiendaire du «prix du Jeune chercheur André Dupont», $\rightarrow) \mathrm{m} / \mathrm{s} 2007$, $n^{\circ} 1$, p. 81 octroyé par le Club de Recherches cliniques du Québec en 2005, fait la synthèse des travaux de son équipe et d'autres études qui défient la notion historique voulant que la sténose aortique soit une maladie dégénérative, donc inévitable. Une association étroite entre le syndrome métabolique et l'accélération de la dégénérescence de la valve aortique a été mise en évidence dans des modèles animaux soumis à un régime riche en graisses et en sucres. Les grandes études prospectives randomisées actuellement en cours permettront sans doute de réconcilier les résultats contradictoires obtenus lors des premiers essais cliniques d'interventions médicamenteuses dans les maladies valvulaires, et il y a tout lieu de croire que le traitement pharmacologique jouera un rôle important dans l'avenir. Si les promesses de la pharmacogénomique se réalisent, il serait peut-être possible d'identifier les patients chez lesquels un processus athérosclérotique attaque aussi la valve ou ceux chez qui un défaut génétique entraîne une ossification 
anormale des feuillets. Un deuxième point fort des études de Pibarot et al. [6] est l'intégration conséquente de toutes les composantes mécaniques dans l'équation diagnostique et thérapeutique, en passant du débit transvalvulaire et de I'hypertension artérielle jusqu'aux considérations géométriques permettant d'éviter la disproportion patient-prothèse. L'intérêt que les maladies valvulaires ont suscité pendant la dernière décennie s'est aussi traduit en une augmentation des lignes directrices de l'American Heart Association (AHA) ACC) pour le suivi des patients atteints de maladies valvulaires: le recueil est passé de 97 pages en 1998 à 147 pages dans l'édition la plus récente en 2006 [7] ! Un emploi plus conséquent des tests à l'effort et à la dobutamine, afin de mieux déceler des situations de bas débit-bas gradient et d'éviter la sous-estimation de la sévérité de la sténose, est recommandé dans les cas de rétrécissement aortique.

\section{Quo vadis?}

Malgré sa simplicité apparente, la valve aortique n'a toujours pas révélé tous ses secrets. Les cas de sténose aortique augmenteront fort certainement au cours des années à venir. Afin de relever le défi, il est impératif de jumeler les études épidémiologiques et mécaniques à des recherches au niveau moléculaire. Des esquisses de Léonard de Vinci jusqu'au premier remplacement valvulaire aortique de Harken en 1960 [8], 450 ans se sont écoulés. À la lumière des progrès récents en épidémiologie, génétique et ingénierie, on peut être optimiste et croire que ces connaissances nouvelles permettront dans un proche avenir le développement d'outils cliniques indispensables. $\diamond$

The aortic valve did not reveal all its secrets

\section{RÉFÉRENCES}

1. Northrup WF $3^{\text {rd }}$, DuBois KA, Kshettry VR, et al. Trends in aortic valve surgery in a large multi-surgeon, multi-hospital practice, 1979-1999. J Heart Valve Dis $2002 ; 11: 768-78$.

2. Roberts WC, Ko JM. Frequency by decades of unicuspid, bicuspid, and tricuspid aortic valves in adults having isolated aortic valve replacement for aortic stenosis, with or without associated aortic regurgitation. Circulation 2005 ; $111: 920-5$.

3. Gharib M, Kremers D, Koochesfahani MM, Kemp M. Leonardo's vision of flow visualization. Exp Fluids $2002 ; 33: 219-23$.

4. Robicsek F. Leonardo da Vinci and the sinuses of Valsalva. Ann Thorac Surg 1991; $52: 328-35$.

5. Garg V, Muth AN, Ransom JF, et al. Mutations in Notchl cause aortic valve disease. Nature $2005 ; 437: 270-4$.

6. Pibarot $P$, Dumesnil JG, Mathieu P. Nouveautés sur le rétrécissement valvulaire aortique. Med Sci (Paris) 2007 ; $23: 81-7$.

7. Bonow RO, Carabello BA, Chatterjee K, et al. ACC/AHA 2006 guidelines for the management of patients with valvular heart disease. J Am Coll Cardiol $2006 ; 48:$ el-148.

8. Harken DE, Soroff HS, Taylor WJ, et al. Partial and complete prostheses in aortic insufficiency. J Thorac Cardiovasc Surg $1960 ; 40:$ 744-62.

\section{TIRÉS À PART}

G. Andelfinger
La Fondation Internationale Balzan

est heureuse de vous présenter les lauréats des

\section{Prix Balzan 2006}

Ludwig Finscher (Allemagne)

pour l'histoire de la musique occidentale depuis le $17^{e}$ siècle

Quentin Skinner (Royaume-Uni)

pour l'histoire et la théorie de la pensée politique

Paolo de Bernardis (Italie) et Andrew Lange (États-Unis) pour l'astronomie et l'astrophysique observationnelles Elliot Meyerowitz (États-Unis)

et Christopher Somerville (États-Unis/ Canada) pour la génétique moléculaire des plantes

La cérémonie de remise des Prix Balzan, qui a lieu alternativement à Rome et à Berne pour respecter le caractère italo-suisse de la Fondation Balzan, s'est tenue cette année à Rome, le vendredi 24 novembre 2006, à l'Accademia dei Lincei, en présence du Président de la République italienne, M. Giorgio Napolitano.

Les Prix Balzan: Le montant de chaque Prix annuel est d'un million de francs suisses (environ 630000 euros), dont la moitié doit être destinée par le lauréat à des projets de recherche, publications, diffusion, instrumentation, etc., impliquant de préférence de jeunes chercheurs.

\section{Prix Balzan 2007}

Les prochains Prix Balzan, d'un million de francs suisses chacun, seront décernés dans les disciplines suivantes:

- littérature européenne (1000-1500)

- droit international après 1945

- immunité innée

- nanoscience

Les candidatures - en provenance d'universités ou d'institutions - devront parvenir au Comité Général Prix de la Fondation Internationale Balzan le $\mathbf{1 5}$ mars 2007 au plus tard.

Documentation demandée :

- un exposé spécifiant l'œuvre et les publications sur lesquelles la proposition se fonde

- une bibliographie

- un curriculum vitae contenant date et lieu de naissance, nationalité, résidence et adresse, une photographie récente, charge actuelle, principales activités académiques

Les candidatures personnelles ne sont pas admises.

Comité Général des Prix

Président Sergio Romano

Fondation Internationale Balzan « Prix "

Piazzetta U. Giordano 4, I-20122 Milano, Italie

Tél. +39 02-7600.2212, Fax +39 02-7600.9457

e-mail: balzan@balzan.it

But et organisation de la Fondation Balzan

La Fondation Internationale Balzan possède deux sièges, l'un à Milan de droit italien, l'autre à Zurich de droit suisse.

La Fondation Internationale Prix E. Balzan "Prix », dont le siège est à Milan, a pour but d'encourager, sans distinction de nationalité, de race et de religion, la culture, les sciences et les initiatives humanitaires les plus méritoires dans le domaine de la paix et de la fraternité entre les peuples. Elle le fait en attribuant chaque année quatre prix d'une valeur totale de quatre millions de francs suisses (environ 2500000 euros). Tous les trois ans - délai minimum - la Fondation Balzan décerne également un prix pour l'humanité, la paix et la fraternité entre les peuples. Les candidatures pour les prix annuels sont présentées par des institutions culturelles et scientifiques du monde entier expressément interpellées à cet effet, et sont ensuite sélectionnées par le Comité Général des Prix, organe de la Fondation dont les 20 membres représentent différents pays européens.

La Fondation Internationale Prix E. Balzan - «Fonds », dont le siège est à Zurich, partage ces objectifs et administre le patrimoine légué par Eugenio Balzan.

Pour plus d'informations : e-mail: balzan@balzan.it - http://www.balzan.it/ 